\title{
BATCH ARRIVAL RETRIAL QUEUING SYSTEM WITH STATE DEPENDENT ADMISSION AND BERNOULLI VACATION
}

\author{
J. Ebenesar Anna Bagyam ${ }^{1}$, K. Udaya Chandrika ${ }^{2}$ \\ ${ }^{1}$ Assistant Professor, Department of Mathematics, SNS College of Technology, Coimbatore, India, \\ ${ }^{2}$ Professor, Department of Mathematics, Avinashilingam Deemed University for Women, Tamil Nadu, India, \\ ebenesar.j@gmail.com, udayachandrika@gmail.com
}

\begin{abstract}
A single server batch arrival retrial queue with server vacation under Bernoulli schedule is considered. Arrivals are controlled according to the state of the server. The necessary and sufficient condition for the system to be stable is derived. Explicit formulae for the stationary distributions and performance measures of the system in steady state are obtained. Numerical examples are presented to illustrate the influence of the parameters on several performance characteristics.
\end{abstract}

Keywords: Retrial queue, batch arrival, state dependent admission control, Bernoulli vacation.

$* * *$

\section{INTRODUCTION}

Retrial queues have the feature that arriving customers finding no free servers must leave the service area and repeat their demands for service after a random time. A customer is said to be in orbit between two retrials. Nowadays, retrial queues have become increasingly important in the analysis of computer and communication networks. For recent papers on retrial queues, see [1], [3], [4], [5] and [13]

In recent years queues with server vacation have emerged as an important area of research due to their various application in production systems, communication systems, computer networks and etc. Some comprehensive studies on the recent results for a variety of vacation models can be found in [6], [7], [10], [11], [12], [14].

In many queuing situations, the customer's arrival rate varies according to the server state idle, busy and on vacation. Altman et al. [2] considered the state dependent M/G/1 type queuing analysis for congestion control in data networks. Madan and Abu-Dayyeh [7] and Madan and Choudhury [8] have investigated classical queuing system with restricted admissibility of arriving batches and Bernoulli server vacation. This paper examines the state dependent retrial queuing system with bulk arrival and server vacation. The similar situation of retrial can be realized in on-line ticket booking centres.

\section{SYSTEM DESCRIPTION}

Consider a single server infinite capacity queuing facility with batch arrival. One of the arriving customers begins his service immediately if the server is available and the remaining customers leave the service area to join the orbit.

The arrival epochs occur in accordance with a Poisson process with rate $\lambda$ and the number of arrivals at each epoch is a random variable $X$ having distribution $P[X=n]=c_{n}$ and moments $\bar{C}_{n}, n \geq 1$. Successive inter retrial times of any customer in orbit is generally distributed with distribution function $\mathrm{A}(\mathrm{x})$ and Laplace transform $\mathrm{A}^{*}(\mathrm{~s})$. The service time is a random variable with distribution function $\mathrm{B}(\mathrm{x})$,

Laplace transform $\mathrm{B}^{*}(\mathrm{~s})$ and finite moments $\mu_{\mathrm{n}}, \mathrm{n} \geq 1$.

After completion of each service, the server may take a vacation with probability $\theta$ or may continue to be in the system with complementary probability. The vacation times are generally distributed with distribution function $\mathrm{V}(\mathrm{x})$,

Laplace transform $\mathrm{V}^{*}(\mathrm{~s})$ and finite moments $\mathrm{v}_{\mathrm{n}}, \mathrm{n} \geq 1$.

The arriving batches are allowed to join the system with state dependent admission control policy. Let $\alpha_{1}, \alpha_{2}$ and $\alpha_{3}$ be the assigned probabilities for an arriving batch to join the system during the period of idle, busy and vacation times respectively.

The hazard rate function of retrial time, service time and vacation time are defined as

$$
\eta(x)=\frac{a(x)}{1-A(x)} ; \mu(x)=\frac{b(x)}{1-B(x)} ; \beta(x)=\frac{v(x)}{1-V(x)} .
$$




\section{THE JOINT DISTRIBUTIONS}

The stage of the system at time $t$ can be described by the Markov process $\left\{(\mathrm{M}(\mathrm{t}) ; \mathrm{t} \geq 0\}=\left\{\mathrm{J}(\mathrm{t}), \mathrm{N}(\mathrm{t}), \xi_{\mathrm{i}}(\mathrm{t}) ; \mathrm{t} \geq 0\right.\right.$, $\mathrm{i}=0, \mathrm{i}=0,1,2\}$ where $\mathrm{J}(\mathrm{t})$ denotes the server state $0,1,2$ according as the server being idle or busy or on vacation and $\mathrm{N}(\mathrm{t})$ denotes the number of customers in the retrial queue at time t. If $J(t)=0$ and $N(t)>0$, then $\xi_{0}(t)$ represents the elapsed retrial time, if $J(t)=1$ and $N(t)>0, \xi_{1}(t)$ corresponds to the elapsed service time of the customer at time 5 , and if $\mathrm{J}(\mathrm{t})=2$ and $\mathrm{N}(\mathrm{t})>0, \xi_{2}(\mathrm{t})$ corresponds to the elapsed vacation time $t$.

For the process $\{\mathrm{M}(\mathrm{t}) ; \mathrm{t} \geq 0\}$, define the probabilities

$\mathrm{I}_{0}(\mathrm{t})=\mathrm{P}\{\mathrm{J}(\mathrm{t})=0, \mathrm{~N}(\mathrm{t})=0\}$

$\mathrm{I}_{\mathrm{n}}(\mathrm{t}, \mathrm{x}) \mathrm{dx}=\mathrm{P}\left\{\mathrm{J}(\mathrm{t})=0, \mathrm{~N}(\mathrm{t})=\mathrm{n}, \mathrm{x} \leq \xi_{0}(\mathrm{t}) \leq \mathrm{x}+\mathrm{dx}\right\}, \mathrm{n} \geq 1$

$\mathrm{W}_{\mathrm{n}}(\mathrm{t}, \mathrm{x}) \mathrm{dx}=\mathrm{P}\left\{\mathrm{J}(\mathrm{t})=1, \mathrm{~N}(\mathrm{t})=\mathrm{n}, \mathrm{x} \leq \xi_{1}(\mathrm{t}) \leq \mathrm{x}+\mathrm{dx}\right\}, \mathrm{n} \geq 0$

$V_{n}(t, x) d x=P\left\{J(t)=2, N(t)=n, x \leq \xi_{2}(t) \leq x+d x\right\}, n \geq 0$

Let $I_{0}, I_{n}(x), W_{n}(x)$ and $V_{n}(x)$ are the limiting densities of $\mathrm{I}_{0}(\mathrm{t}), \mathrm{I}_{\mathrm{n}}(\mathrm{t}, \mathrm{x}), \mathrm{W}_{\mathrm{n}}(\mathrm{t}, \mathrm{x})$ and $\mathrm{V}_{\mathrm{n}}(\mathrm{t}, \mathrm{x})$.

Define the probability generating function

$\mathrm{I}(\mathrm{z}, \mathrm{x})=\sum_{\mathrm{n}=1}^{\infty} \mathrm{I}_{\mathrm{n}}(\mathrm{x}) \mathrm{z}^{\mathrm{n}} ; \mathrm{W}(\mathrm{z}, \mathrm{x})=\sum_{\mathrm{n}=0}^{\infty} \mathrm{W}_{\mathrm{n}}(\mathrm{x}) \mathrm{z}^{\mathrm{n}}$

$V(z, x)=\sum_{n=1}^{\infty} V_{n}(x) z^{n}$ and $c(z)=\sum_{n=1}^{\infty} c_{n} z^{n}$

\section{ERGODICITY CONDITION}

Let $\left\{\tau_{n}, n \in N\right\}$ be the sequence of epochs of the service completion times or vacation termination times. The sequence of random vectors $\mathrm{Q}_{\mathrm{n}}=\left\{\mathrm{J}\left(\tau_{\mathrm{n}}^{+}\right), \mathrm{N}\left(\tau_{\mathrm{n}}^{+}\right)\right\}$forms a Markov chain, which is the embedded Markov chain for our queuing system with state space $S=\{0,1,2\} \times\{0,1,2, \ldots\}$

\section{Theorem1}

$\left\{\mathrm{Q}_{\mathrm{n}}, \mathrm{n} \geq 1\right\}$ is ergodic if and only if $\overline{\mathrm{C}}_{1}\left[1-\mathrm{A}^{*}\left(\alpha_{1} \lambda\right)\right]+\lambda \overline{\mathrm{C}}_{1}\left[\alpha_{2} \mu_{1}+\alpha_{3} \theta \mathrm{v}_{1}\right]<1$

\section{Proof}

$\left\{\mathrm{Q}_{\mathrm{n}}, \mathrm{n} \geq 1\right\}$ is an irreducible and aperiodic Markov chain. To prove ergodicity, we shall use Foster's criterion : An irreducible and aperiodic Markov chain is ergodic if there exists a non negative function $f(j), j \in N$ and $\varepsilon>0$ such that the mean drift $\Psi_{j}=E\left[f\left(Q_{n+1}\right)-f\left(Q_{n}\right) \mid Q_{n}=j\right]$ is finite for all $\mathrm{j} \in \mathrm{N}$ and $\Psi_{\mathrm{j}} \leq-\varepsilon$ for all $\mathrm{j} \in \mathrm{N}$, except perhaps a finite number.

Take $f(j)=j$. Then we have

$\Psi_{j}= \begin{cases}\overline{\mathrm{C}}_{1}\left[1-\mathrm{A}^{*}\left(\alpha_{1} \lambda\right)\right]+\lambda \overline{\mathrm{C}}_{1}\left[\alpha_{2} \mu_{1}+\alpha_{3} \theta \mathrm{v}_{1}\right]-1 & \text { if } \mathrm{j}=1,2,3, \ldots \\ \lambda \overline{\mathrm{C}}_{1}\left[\alpha_{2}(1-\theta)+\alpha_{3} \theta \mathrm{v}_{1}\right]-1 & \text { if } \mathrm{j}=0\end{cases}$

Clearly,

the

inequality

$\overline{\mathrm{C}}_{1}\left[1-\mathrm{A}^{*}\left(\alpha_{1} \lambda\right)\right]+\lambda \overline{\mathrm{C}}_{1}\left[\alpha_{2} \mu_{1}+\alpha_{3} \theta \mathrm{v}_{1}\right]<1 \quad$ is a sufficient condition for ergodicity. The same inequality is also necessary for ergodicity. We can guarantee the non-ergodicity of the Markov chain $\left\{Q_{n}, n \geq 1\right\}$, if it satisfies Kaplan's condition, namely $\Psi_{\mathrm{j}}<\infty$ for all $\mathrm{j} \in \mathrm{N}$ and there exists $\mathrm{j}_{0} \in \mathrm{N}$ such that $\Psi_{\mathrm{j}} \geq 0$ for $\mathrm{j} \geq \mathrm{j}_{0}$. In our case, Kaplan's condition is satisfied because there exists $k \in N$ such that $r_{i j}=0$ for $j<i-k$ and $\mathrm{i}>0$, where $\mathrm{R}=\left(\mathrm{r}_{\mathrm{ij}}\right)$ is the one step transition matrix of $\left\{\mathrm{Q}_{\mathrm{n}}, \mathrm{n} \geq 1\right\}$. Then the inequality $\overline{\mathrm{C}}_{1}\left[1-\mathrm{A}^{*}\left(\alpha_{1} \lambda\right)\right]+\lambda \overline{\mathrm{C}}_{1}\left[\alpha_{2} \mu_{1}+\alpha_{3} \theta \mathrm{v}_{1}\right]<1$ implies the non ergodicity of the Markov chain.

Since the arrival stream is a Poisson process, it can be shown from Burke's theorem that the steady state probabilities of $\{\mathrm{J}(\mathrm{t}), \mathrm{N}(\mathrm{t}), \mathrm{t} \geq 0\}$ exist and are positive if and only if $\overline{\mathrm{C}}_{1}\left[1-\mathrm{A}^{*}\left(\alpha_{1} \lambda\right)\right]+\lambda \overline{\mathrm{C}}_{1}\left[\alpha_{2} \mu_{1}+\alpha_{3} \theta \mathrm{v}_{1}\right]<1$.

From the mean drift $\Psi_{\mathrm{j}}=$ $\overline{\mathrm{C}}_{1}\left[1-\mathrm{A}^{*}\left(\alpha_{1} \lambda\right)\right]+\lambda \overline{\mathrm{C}}_{1}\left[\alpha_{2} \mu_{1}+\alpha_{3} \theta \mathrm{v}_{1}\right]-1$, for $\mathrm{j} \geq 1$ we have the reasonable conclusion that the term $\lambda \overline{\mathrm{C}}_{1}\left[\alpha_{2} \mu_{1}+\alpha_{3} \theta \mathrm{v}_{1}\right]$ represents a batch arrival during service time and on vacation time. The other term $\overline{\mathrm{C}}_{1}\left[1-\mathrm{A}^{*}\left(\alpha_{1} \lambda\right)\right]-1$ refers to the contribution to the orbit size due to batch arrival during the retrial time excluding the arbitrary customer of the arriving batch whose service commences so that he no longer belongs to the orbit. Similar interpretation can be provided for $j=0$. the condition $\Psi_{\mathrm{j}}<0$ assures that the orbit size does not grow indefinitely in course of time. 


\section{STEADY STATE PROBABILITY GENERATION FUNCTION}

The steady state equations that governs the system under consideration are

$\lambda \mathrm{I}_{0} \quad=\int_{0}^{\infty} \mathrm{V}_{0}(\mathrm{x}) \beta(\mathrm{x}) \mathrm{dx}+(1-\theta) \int_{0}^{\infty} \mathrm{W}_{0}(\mathrm{x}) \mu(\mathrm{x}) \mathrm{dx}$

$\frac{d}{d x} I_{n}(x)=-(\lambda+\eta(x)) I_{n}(x)+\lambda\left(1-\alpha_{1}\right) I_{n}(x), n \geq 1$

$\frac{\mathrm{d}}{\mathrm{dx}} \mathrm{W}_{\mathrm{n}}(\mathrm{x})=-(\lambda+\mu(\mathrm{x})) \mathrm{W}_{\mathrm{n}}(\mathrm{x})+\lambda\left(1-\alpha_{2}\right) \mathrm{W}_{\mathrm{n}}(\mathrm{x})$

$$
+\lambda \alpha_{2}\left(1-\delta_{0 \mathrm{n}}\right) \sum_{\mathrm{k}=1}^{\mathrm{n}} \mathrm{c}_{\mathrm{k}} \mathrm{W}_{\mathrm{n}-\mathrm{k}}(\mathrm{x}), \mathrm{n} \geq 0(3)
$$

$\frac{\mathrm{d}}{\mathrm{dx}} \mathrm{V}_{\mathrm{n}}(\mathrm{x})=-(\lambda+\beta(\mathrm{x})) \mathrm{V}_{\mathrm{n}}(\mathrm{x})+\lambda\left(1-\alpha_{3}\right) \mathrm{V}_{\mathrm{n}}(\mathrm{x})$

$$
+\lambda \alpha_{3}\left(1-\delta_{0 \mathrm{n}}\right) \sum_{\mathrm{k}=1}^{\mathrm{n}} \mathrm{c}_{\mathrm{k}} \mathrm{V}_{\mathrm{n}-\mathrm{k}}(\mathrm{x}), \mathrm{n} \geq 0
$$

With boundary conditions

$I_{n}(0)=\int_{0}^{\infty} V_{n}(x) \beta(x) d x+(1-\theta) \int_{0}^{\infty} W_{n}(x) \mu(x) d x, n \geq 1(5)$

$\mathrm{W}_{0}(0)=\lambda \mathrm{c}_{1} \mathrm{I}_{0}+\int_{0}^{\infty} \mathrm{I}_{1}(\mathrm{x}) \eta(\mathrm{x}) \mathrm{dx}$

$\mathrm{W}_{\mathrm{n}}(0)=\lambda \mathrm{c}_{\mathrm{n}+1} \mathrm{I}_{0}+\int_{0}^{\infty} \mathrm{I}_{\mathrm{n}+1}(\mathrm{x}) \eta(\mathrm{x}) \mathrm{dx}$

$$
+\lambda \alpha_{1} \int_{0}^{\infty} \sum_{\mathrm{k}=1}^{\mathrm{n}} \mathrm{c}_{\mathrm{k}} \mathrm{I}_{\mathrm{n}-\mathrm{k}+1}(\mathrm{x}) \mathrm{dx}, \mathrm{n} \geq 1
$$

$\mathrm{V}_{\mathrm{n}}(0)=\theta \int_{0}^{\infty} \mathrm{W}_{\mathrm{n}}(\mathrm{x}) \mu(\mathrm{x}) \mathrm{dx}, \mathrm{n} \geq 0$

From the equations (2) - (8) we have,

$$
\begin{aligned}
& \mathrm{I}(\mathrm{z}, \mathrm{x})=\mathrm{I}(\mathrm{z}, 0) \mathrm{e}^{-\alpha_{1} \lambda \mathrm{x}}[1-\mathrm{A}(\mathrm{x})] \\
& \mathrm{W}(\mathrm{z}, \mathrm{x})=\mathrm{W}(\mathrm{z}, 0) \mathrm{e}^{-\alpha_{2} \lambda(1-\mathrm{c}(\mathrm{z})) \mathrm{x}}[1-\mathrm{B}(\mathrm{x})] \\
& \mathrm{V}(\mathrm{z}, \mathrm{x})=\mathrm{V}(\mathrm{z}, 0) \mathrm{e}^{-\alpha_{3} \lambda(1-\mathrm{c}(\mathrm{z})) \mathrm{x}}[1-\mathrm{V}(\mathrm{x})]
\end{aligned}
$$

$$
\begin{aligned}
\mathrm{I}(z, 0)= & \int_{0}^{\infty} \mathrm{V}(\mathrm{z}, \mathrm{x}) \beta(\mathrm{x}) \mathrm{dx} \\
& +(1-\theta) \int_{0}^{\infty} \mathrm{W}(\mathrm{z}, \mathrm{x}) \mu(\mathrm{x}) \mathrm{dx}-\lambda \mathrm{I}_{0} \\
\mathrm{~W}(\mathrm{z}, 0)= & \mathrm{I}(\mathrm{z}, 0)\left[\mathrm{A}^{*}\left(\alpha_{1} \lambda\right)+\mathrm{c}(\mathrm{z})\left(1-\mathrm{A}^{*}\left(\alpha_{1} \lambda\right)\right)\right] \\
& +\lambda \mathrm{I}_{0} \mathrm{c}(\mathrm{z})
\end{aligned}
$$

Substituting the expressions of $\mathrm{V}(\mathrm{z}, \mathrm{x})$ and $\mathrm{W}(\mathrm{z}, \mathrm{x})$ in terms of $\mathrm{W}(\mathrm{z}, 0)$ in equation $(12)$, we get

$$
\begin{aligned}
\mathrm{I}(\mathrm{z}, 0)= & \theta \mathrm{W}(\mathrm{z}, 0) \mathrm{B}^{*}\left(\alpha_{2} \lambda(1-\mathrm{c}(\mathrm{z}))\right) \mathrm{V}^{*}\left(\alpha_{3} \lambda(1-\mathrm{c}(\mathrm{z}))\right) \\
& +(1-\theta) \mathrm{W}(\mathrm{z}, 0) \mathrm{B}^{*}\left(\alpha_{2} \lambda(1-\mathrm{c}(\mathrm{z}))\right)-\lambda \mathrm{I}_{0} \\
= & \mathrm{W}(\mathrm{z}, 0) \mathrm{B}^{*}\left(\alpha_{2} \lambda(1-\mathrm{c}(\mathrm{z}))\right) \\
& {\left[1-\theta+\theta \mathrm{V}^{*}\left(\alpha_{3} \lambda(1-\mathrm{c}(\mathrm{z}))\right)\right]-\lambda \mathrm{I}_{0} }
\end{aligned}
$$

Using the expression $\mathrm{I}(\mathrm{z}, 0)$ in equation (13) and simplifying we obtain

$\mathrm{W}(\mathrm{z}, 0)=\lambda \mathrm{I}_{0} \mathrm{~A}^{*}\left(\alpha_{1} \lambda\right)[1-\mathrm{c}(\mathrm{z})] / \mathrm{D}(\mathrm{z})$

Where

$\mathrm{D}(\mathrm{z})=\mathrm{B}^{*}\left(\alpha_{2} \lambda(1-\mathrm{c}(\mathrm{z}))\right)\left[1-\theta+\theta \mathrm{V}^{*}\left(\alpha_{3} \lambda(1-\mathrm{c}(\mathrm{z}))\right)\right]$

$$
\left[\mathrm{A}^{*}\left(\alpha_{1} \lambda\right)+\mathrm{c}(\mathrm{z})\left(1-\mathrm{A}^{*}\left(\alpha_{1} \lambda\right)\right)\right]-\mathrm{z}
$$

Now equations (15) and (14) become

$$
\begin{aligned}
\mathrm{I}(z, 0)= & \lambda \mathrm{I}_{0}\left\{\mathrm{z}-\mathrm{c}(\mathrm{z}) \mathrm{B}^{*}\left(\alpha_{2} \lambda(1-\mathrm{c}(\mathrm{z}))\right)\right. \\
& {\left.\left[1-\theta+\theta \mathrm{V}^{*}\left(\alpha_{3} \lambda(1-\mathrm{c}(\mathrm{z}))\right)\right]\right\} / \mathrm{D}(\mathrm{z}) } \\
\mathrm{V}(\mathrm{z}, 0)= & \lambda \mathrm{I}_{0} \mathrm{~A}^{*}\left(\alpha_{1} \lambda\right)[1-\mathrm{c}(\mathrm{z})] \theta \mathrm{B}^{*}\left(\alpha_{2} \lambda(1-\mathrm{c}(\mathrm{z}))\right) \\
& / \mathrm{D}(\mathrm{z})
\end{aligned}
$$

\section{Theorem 2}

Using equilibrium state, the joint distribution of the server has the following partial generating functions

$$
\begin{aligned}
\mathrm{I}(\mathrm{z})= & \mathrm{I}_{0}\left[\mathrm{z}-\mathrm{c}(\mathrm{z}) \mathrm{B}^{*}\left(\alpha_{2} \lambda(1-\mathrm{c}(\mathrm{z}))\right)\left[1-\mathrm{A}^{*}\left(\alpha_{1} \lambda\right)\right]\right. \\
& {\left.\left[1-\theta+\theta \mathrm{V}^{*}\left(\alpha_{3} \lambda(1-\mathrm{c}(\mathrm{z}))\right)\right]\right\} /\left[\alpha_{1} \mathrm{D}(\mathrm{z})\right](20) }
\end{aligned}
$$




$$
\begin{aligned}
\mathrm{V}(\mathrm{z}) & =\mathrm{I}_{0} \mathrm{~A}^{*}\left(\alpha_{1} \lambda\right) \theta \mathrm{B}^{*}\left(\alpha_{2} \lambda(1-\mathrm{c}(\mathrm{z}))\right) \\
& {\left.\left[1-\mathrm{V}^{*}\left(\alpha_{3} \lambda(1-\mathrm{c}(\mathrm{z}))\right)\right]\right\} /\left[\alpha_{3} \mathrm{D}(\mathrm{z})\right](22) } \\
\mathrm{I}_{0} & =\alpha_{1} \mathrm{~T}_{1} / \mathrm{T}_{2}
\end{aligned}
$$

Where

$$
\begin{aligned}
\mathrm{T}_{1}= & 1-\overline{\mathrm{C}}_{1}\left[1-\mathrm{A}^{*}\left(\alpha_{1} \lambda\right)\right]-\lambda \overline{\mathrm{C}}_{1}\left[\alpha_{2} \mu_{1}+\alpha_{3} \theta \mathrm{v}_{1}\right] \\
\mathrm{T}_{2}= & \alpha_{1} \mathrm{~T}_{1}+\left(1-\mathrm{A}^{*}\left(\alpha_{1} \lambda\right)\left(\mathrm{A}^{*}\left(\alpha_{1} \lambda\right) \overline{\mathrm{C}}_{1}-\mathrm{T}_{1}\right)\right. \\
& +\alpha_{1} \mathrm{~A}^{*}\left(\alpha_{1} \lambda\right) \lambda \overline{\mathrm{C}}_{1}\left[\mu_{1}+\theta \mathrm{v}_{1}\right]
\end{aligned}
$$

\section{Proof}

Substituting for $\mathrm{I}(\mathrm{z}, 0), \mathrm{W}(\mathrm{z}, 0)$ and $\mathrm{V}(\mathrm{z}, \mathrm{x})$ given by equations (9), (10) and (11) and integrating with respect to $\mathrm{x}$ from 0 to $\infty$ we get the results given in equations (20) - (22).

Now, the unknown constant $I_{0}$ given in equation (23) can be determined by using the normalizing condition $\mathrm{I}_{0}+\mathrm{I}(1)+\mathrm{W}(1)+\mathrm{V}(1)=1$.

\section{MEAN ORBIT SIZE AND MEAN SYSTEM SIZE}

\section{Theorem 3}

The probability generating function of the number of customer in the orbit is

$$
\begin{aligned}
\mathrm{P}_{\mathrm{q}}(\mathrm{z})= & \mathrm{I}_{0}\left[\theta \alpha_{1} \alpha_{2}\left(1-\alpha_{3}\right) \mathrm{A}^{*}\left(\alpha_{1} \lambda\right) \mathrm{B}^{*}\left(\alpha_{2} \lambda(1-\mathrm{c}(\mathrm{z}))\right)\right. \\
& {\left[1-\mathrm{V}^{*}\left(\alpha_{3} \lambda(1-\mathrm{c}(\mathrm{z}))\right)\right]-\alpha_{1}\left(1-\alpha_{2}\right) \alpha_{3} } \\
& \mathrm{~B}^{*}\left(\alpha_{2} \lambda(1-\mathrm{c}(\mathrm{z}))\right) \mathrm{A}^{*}\left(\alpha_{1} \lambda\right) \\
& -\left(1-\alpha_{1}\right) \alpha_{2} \alpha_{3}\left[1-\mathrm{A}^{*}\left(\alpha_{1} \lambda\right)\right] \mathrm{c}(\mathrm{z}) \\
& \mathrm{B}^{*}\left(\alpha_{2} \lambda(1-\mathrm{c}(\mathrm{z}))\right)\left[1-\theta+\theta \mathrm{V}^{*}\left(\alpha_{3} \lambda(1-\mathrm{c}(\mathrm{z}))\right)\right] \\
& +\alpha_{1} \alpha_{3} \mathrm{~A}^{*}\left(\alpha_{1} \lambda\right) \\
& \left.+\alpha_{2} \alpha_{3} \mathrm{z}\left(1-\alpha_{1}-\mathrm{A}^{*}\left(\alpha_{1} \lambda\right)\right)\right\} /\left[\alpha_{1} \alpha_{2} \alpha_{3} \mathrm{D}(\mathrm{z})\right]
\end{aligned}
$$

The probability generating function of number of customer in the system is

$$
\begin{aligned}
\mathrm{P}_{\mathrm{q}}(\mathrm{z})= & \mathrm{I}_{0}\left[\theta \alpha_{1} \alpha_{2}\left(1-\alpha_{3}\right) \mathrm{A}^{*}\left(\alpha_{1} \lambda\right) \mathrm{B}^{*}\left(\alpha_{2} \lambda(1-\mathrm{c}(\mathrm{z}))\right)\right. \\
& {\left[1-\mathrm{V}^{*}\left(\alpha_{3} \lambda(1-\mathrm{c}(\mathrm{z}))\right)\right] } \\
& -\alpha_{1} \alpha_{2} \alpha_{3} \mathrm{~A}^{*}\left(\alpha_{1} \lambda\right) \mathrm{B}^{*}\left(\alpha_{2} \lambda(1-\mathrm{c}(\mathrm{z}))\right) \\
& -\left(1-\alpha_{1}\right) \alpha_{2} \alpha_{3}\left(1-\mathrm{A}^{*}\left(\alpha_{1} \lambda\right)\right) \\
& \mathrm{c}(\mathrm{z}) \mathrm{B}^{*}\left(\alpha_{2} \lambda(1-\mathrm{c}(\mathrm{z}))\right)
\end{aligned}
$$

$$
\begin{aligned}
& {\left[1-\theta+\theta \mathrm{V}^{*}\left(\alpha_{3} \lambda(1-\mathrm{c}(\mathrm{z}))\right)\right]} \\
& +\left(1-\alpha_{1}\right) \alpha_{2} \alpha_{3} \mathrm{z} \mathrm{A}^{*}\left(\alpha_{1} \lambda\right) \\
& \left.\left(\alpha_{1}-\alpha_{2}-\alpha_{1} \mathrm{~B}^{*}\left(\alpha_{2} \lambda(1-\mathrm{c}(\mathrm{z}))\right)\right)\right\} \\
& /\left[\alpha_{1} \alpha_{2} \alpha_{3} \mathrm{D}(\mathrm{z})\right]
\end{aligned}
$$

\section{Proof}

The probability generating function for the number of customer in the orbit is

$\mathrm{P}_{\mathrm{q}}(\mathrm{z})=\mathrm{I}_{0}+\mathrm{I}(\mathrm{z})+\mathrm{W}(\mathrm{z})+\mathrm{V}(\mathrm{z})$ and

The probability generating function for the number of customer in the system is

$\mathrm{P}_{\mathrm{s}}(\mathrm{z})=\mathrm{I}_{0}+\mathrm{I}(\mathrm{z})+\mathrm{zW}(\mathrm{z})+\mathrm{V}(\mathrm{z})$

Substituting the expressions of $\mathrm{I}(\mathrm{z}), \mathrm{W}(\mathrm{z})$ and $\mathrm{V}(\mathrm{z})$, we get the equations as in (24) and (25).

\section{Corollary 1}

The mean number of customer in the orbit is

$$
\mathrm{L}_{\mathrm{q}}=\mathrm{N}_{2} / \mathrm{T}_{2}+\mathrm{N}_{1} \mathrm{~T}_{3} /\left(\mathrm{T}_{1} \mathrm{~T}_{2}\right)
$$

The mean number of customer in the system is

$$
\mathrm{L}_{\mathrm{s}}=\mathrm{L}_{\mathrm{q}}+\mathrm{A}^{*}\left(\alpha_{1} \lambda\right) \alpha_{1} \lambda \overline{\mathrm{C}}_{1} \mu_{1} / \mathrm{T}_{2}
$$

Where

$$
\begin{aligned}
\mathrm{T}_{3}= & \lambda^{2} \overline{\mathrm{C}}_{1}^{2} \alpha_{2} \alpha_{3} \theta \mu_{1} \mathrm{v}_{1}+\lambda \overline{\mathrm{C}}_{1}^{2} \alpha_{2} \mu_{1}\left(1-\mathrm{A}^{*}\left(\alpha_{1} \lambda\right)\right) \\
+ & \lambda \overline{\mathrm{C}}_{1}^{2} \alpha_{3} \theta \mathrm{v}_{1}\left(1-\mathrm{A}^{*}\left(\alpha_{1} \lambda\right)\right)+\left[\lambda^{2} \alpha_{2}^{2} \overline{\mathrm{C}}_{1}^{2} \mu_{2}\right. \\
& \left.+\theta \alpha_{3}^{2} \lambda^{2} \overline{\mathrm{C}}_{1}^{2} \mathrm{v}_{2}+\overline{\mathrm{C}}_{2}\left(1-\mathrm{A}^{*}\left(\alpha_{1} \lambda\right)\right)\right] / 2 \\
\mathrm{~N}_{1}= & \left(1-\alpha_{1}\right)\left(1-\mathrm{A}^{*}\left(\alpha_{1} \lambda\right)\right)\left[\overline{\mathrm{C}}_{1}+\alpha_{2} \lambda \overline{\mathrm{C}}_{1} \mu_{1}\right. \\
+ & \left.\alpha_{3} \lambda \theta \overline{\mathrm{C}}_{1} \mathrm{v}_{1}\right]-\left(1-\alpha_{1}-\mathrm{A}^{*}\left(\alpha_{1} \lambda\right)\right) \\
& +\alpha_{1} \lambda \overline{\mathrm{C}}_{1} \mathrm{~A}^{*}\left(\alpha_{1} \lambda\right)\left[\theta\left(1-\alpha_{3}\right) \mathrm{v}_{1}+\left(1-\alpha_{2}\right) \mu_{1}\right] \\
\mathrm{N}_{2}= & \alpha_{1}\left(1-\alpha_{3}\right) \theta \mathrm{A}^{*}\left(\alpha_{1} \lambda\right) \alpha_{2} \lambda^{2} \overline{\mathrm{C}}_{1}^{2} \mu_{1} \mathrm{v}_{1} \\
& +\left\{\alpha_{1} \alpha_{3}\left(1-\alpha_{3}\right) \theta \mathrm{A}^{*}\left(\alpha_{1} \lambda\right) \lambda^{2} \overline{\mathrm{C}}_{1}^{2} \mathrm{v}_{2}\right. \\
& +\alpha_{1} \alpha_{2}\left(1-\alpha_{2}\right) \mathrm{A}^{*}\left(\alpha_{1} \lambda\right) \lambda^{2} \overline{\mathrm{C}}_{1}^{2} \mu_{2} \\
& +\left(1-\alpha_{1}\right)\left(1-\mathrm{A}^{*}\left(\alpha_{1} \lambda\right)\right)\left[\overline{\mathrm{C}}_{2}+\alpha_{2}^{2} \lambda^{2} \overline{\mathrm{C}}_{1}^{2} \mu_{2}\right. \\
& \left.\left.+\theta \alpha_{3}^{2} \lambda^{2} \overline{\mathrm{C}}_{1}^{2} \mathrm{v}_{2}\right]\right\} / 2+\left(1-\alpha_{1}\right)\left(1-\mathrm{A}^{*}\left(\alpha_{1} \lambda\right)\right)
\end{aligned}
$$




$$
\begin{aligned}
& {\left[\alpha_{2} \lambda \overline{\mathrm{C}}_{1}^{2} \mu_{1}+\alpha_{3} \lambda \theta \overline{\mathrm{C}}_{1}^{2} \mathrm{v}_{1}\right.} \\
& \left.+\theta \alpha_{3} \alpha_{2} \lambda^{2} \overline{\mathrm{C}}_{1}^{2} \mu_{1} \mathrm{v}_{1}\right]
\end{aligned}
$$

\section{Proof}

Differentiating $\mathrm{P}_{\mathrm{q}}(\mathrm{z})$ and $\mathrm{P}_{\mathrm{s}}(\mathrm{z})$ with respect to $\mathrm{z}$ and taking limit $\mathrm{z} \rightarrow 1$ by using $\mathrm{L}^{\prime}$ Hospital rule the expressions for $\mathrm{L}_{\mathrm{q}}$ and $\mathrm{L}_{\mathrm{s}}$ can be obtained.

\section{OPERATING CHARACTERISTICS}

Some performance measures for the system are given below.

1. The steady state probability that the server is idle in the empty system is

$$
\mathrm{I}_{0}=\alpha_{1} \mathrm{~T}_{1} / \mathrm{T}_{2}
$$

2. The steady state probability that the server is idle in the non-empty system is

$$
\mathrm{I}=\left[1-\mathrm{A}^{*}\left(\alpha_{1} \lambda\right)\right]\left[\alpha_{2} \lambda \overline{\mathrm{C}}_{1} \mu_{1}+\theta \alpha_{3} \lambda \overline{\mathrm{C}}_{1} \mathrm{v}_{1}+\overline{\mathrm{C}}_{1}-1\right] / \mathrm{T}_{2}
$$

3. The steady state probability that the server is busy is

$$
\mathrm{W}=\alpha_{1} \mathrm{~A}^{*}\left(\alpha_{1} \lambda\right) \lambda \overline{\mathrm{C}}_{1} \mu_{1} / \mathrm{T}_{2}
$$

4. The steady state probability that the server is on vacation is

$$
\mathrm{V}=\alpha_{1} \mathrm{~A}^{*}\left(\alpha_{1} \lambda\right) \theta \lambda \overline{\mathrm{C}}_{1} \mathrm{v}_{1} / \mathrm{T}_{2}
$$

5. The probability that the orbit is empty while the server is busy is

$\mathrm{W}_{0}=\alpha_{1} \mathrm{~T}_{1}\left[1-\mathrm{B}^{*}\left(\alpha_{2} \lambda\right)\right] /\left\{\alpha_{2} \mathrm{~T}_{2} \mathrm{~B}^{*}\left(\alpha_{2} \lambda\right)\left[1-\theta+\theta \mathrm{V}^{*}\left(\alpha_{3} \lambda\right)\right]\right\}$

6. The probability that the orbit is empty while the server is on vacation is

$$
\mathrm{V}_{0}=\theta \alpha_{1} \mathrm{~T}_{1}\left[1-\mathrm{V}^{*}\left(\alpha_{3} \lambda\right)\right] /\left\{\alpha_{3} \mathrm{~T}_{2}\left[1-\theta+\theta \mathrm{V}^{*}\left(\alpha_{3} \lambda\right)\right]\right\}
$$

7. The probability of orbit being empty is

$$
\begin{aligned}
E= & I_{0}+W_{0}+V_{0} \\
= & \alpha_{1} T_{1}\left\{\alpha_{2} \alpha_{3} B^{*}\left(p_{2} \lambda\right)\left[1-\theta+\theta V^{*}\left(\alpha_{3} \lambda\right)\right]\right. \\
& +\alpha_{3}\left[1-B^{*}\left(\alpha_{2} \lambda\right)\right] \\
& +\theta \alpha_{2} B^{*}\left(\alpha_{2} \lambda\right)\left[1-V^{*}\left(\alpha_{3} \lambda\right)\right\}
\end{aligned}
$$

\section{NUMERICAL ILLUSTRATION}

Numerical results are obtained when the service, retrial and vacation times follow exponential distribution. Table shows

\begin{tabular}{|c|c|c|c|c|c|c|c|}
\hline$\lambda$ & $\mu$ & $\eta$ & $\mathrm{I}_{0}$ & I & W & V & $\mathrm{L}_{\mathrm{q}}$ \\
\hline \multirow[t]{12}{*}{1} & \multirow[t]{4}{*}{10} & 20 & 0.7125 & 0.0281 & 0.1297 & 0.1297 & 0.0895 \\
\hline & & 30 & 0.7213 & 0.0188 & 0.1299 & 0.1299 & 0.0748 \\
\hline & & 40 & 0.7258 & 0.0141 & 0.1301 & 0.1301 & 0.0675 \\
\hline & & 50 & 0.7284 & 0.0113 & 0.1301 & 0.1301 & 0.0632 \\
\hline & \multirow[t]{4}{*}{30} & 20 & 0.7920 & 0.0271 & 0.0452 & 0.1356 & 0.0644 \\
\hline & & 30 & 0.8007 & 0.0181 & 0.0453 & 0.1359 & 0.0520 \\
\hline & & 40 & 0.8051 & 0.0136 & 0.0453 & 0.1360 & 0.0459 \\
\hline & & 50 & 0.8077 & 0.0109 & 0.0454 & 0.1361 & 0.0423 \\
\hline & \multirow[t]{4}{*}{50} & 20 & 0.8088 & 0.0269 & 0.0274 & 0.1369 & 0.0608 \\
\hline & & 30 & 0.8175 & 0.0180 & 0.0274 & 0.1371 & 0.0489 \\
\hline & & 40 & 0.8218 & 0.0135 & 0.0274 & 0.1372 & 0.0429 \\
\hline & & 50 & 0.8244 & 0.0108 & 0.0275 & 0.1373 & 0.0394 \\
\hline \multirow[t]{12}{*}{2} & \multirow[t]{4}{*}{10} & 20 & 0.4833 & 0.0608 & 0.2280 & 0.2280 & 0.3565 \\
\hline & & 30 & 0.5015 & 0.0407 & 0.2289 & 0.2289 & 0.3005 \\
\hline & & 40 & 0.5107 & 0.0306 & 0.2294 & 0.2294 & 0.2738 \\
\hline & & 50 & 0.5162 & 0.0245 & 0.2296 & 0.2296 & 0.2581 \\
\hline & \multirow[t]{4}{*}{30} & 20 & 0.6129 & 0.0577 & 0.0824 & 0.2471 & 0.2253 \\
\hline & & 30 & 0.6307 & 0.0386 & 0.0827 & 0.2481 & 0.1868 \\
\hline & & 40 & 0.6396 & 0.0290 & 0.0829 & 0.2486 & 0.1682 \\
\hline & & 50 & 0.6450 & 0.0232 & 0.0829 & 0.2488 & 0.1572 \\
\hline & \multirow[t]{4}{*}{50} & 20 & 0.6414 & 0.0570 & 0.0503 & 0.2513 & 0.2075 \\
\hline & & 30 & 0.6591 & 0.0381 & 0.0505 & 0.2523 & 0.1717 \\
\hline & & 40 & 0.6680 & 0.0286 & 0.0506 & 0.2528 & 0.1543 \\
\hline & & 50 & 0.6734 & 0.0229 & 0.0506 & 0.2531 & 0.1441 \\
\hline \multirow[t]{10}{*}{3} & \multirow[t]{4}{*}{10} & 20 & 0.2949 & 0.0964 & 0.3044 & 0.3044 & 1.0085 \\
\hline & & 30 & 0.3227 & 0.0647 & 0.3063 & 0.3063 & 0.8163 \\
\hline & & 40 & 0.3367 & 0.0487 & 0.3073 & 0.3073 & 0.7308 \\
\hline & & 50 & 0.3451 & 0.0390 & 0.3079 & 0.3079 & 0.6824 \\
\hline & \multirow[t]{4}{*}{30} & 20 & 0.4562 & 0.0906 & 0.1133 & 0.3399 & 0.5360 \\
\hline & & 30 & 0.4833 & 0.0608 & 0.1140 & 0.3419 & 0.4400 \\
\hline & & 40 & 0.4970 & 0.0457 & 0.1143 & 0.3430 & 0.3952 \\
\hline & & 50 & 0.5052 & 0.0367 & 0.1145 & 0.3436 & 0.3693 \\
\hline & \multirow[t]{2}{*}{50} & 20 & 0.4931 & 0.0893 & 0.0696 & 0.3480 & 0.4808 \\
\hline & & 30 & 0.5200 & 0.0599 & 0.0700 & 0.3501 & 0.3956 \\
\hline
\end{tabular}
the dependence of the performance measures $\mathrm{I}_{0}, \mathrm{I}, \mathrm{W}, \mathrm{V}$ and $\mathrm{L}_{\mathrm{q}}$ for the fixed values of $\left(\theta, \beta, \alpha_{1}, \alpha_{2}, \alpha_{3}, \mathrm{c}_{1}, \mathrm{c}_{2}\right)=(5,5$, $0.8,0.5,0.5,0.5,0.5)$. Figures show the effect of $\alpha_{1}, \alpha_{2}$ and $\alpha_{3}$ (joining probability during the period of idle, busy and vacation) on the performance measures. $\mathrm{L}_{\mathrm{q}}$ the mean number of customer in the orbit for the parameters $\left(\lambda, \eta, \mu, \theta, \beta, \alpha_{1}, \alpha_{2}, \alpha_{3}, c_{1}, c_{2}\right)=(2,15,5,5,5,0.8,0.5,0.5$, $0.5,0.5)$.

Table1. Performance measures for the various values of $\lambda, \mu$ and $\eta$ 


\begin{tabular}{|l|l|l|l|l|l|l|l|}
\hline & & 40 & 0.5336 & 0.0451 & 0.0702 & 0.3511 & 0.3556 \\
\cline { 3 - 7 } & 50 & 0.5418 & 0.0361 & 0.0704 & 0.3518 & 0.3324 \\
\hline
\end{tabular}

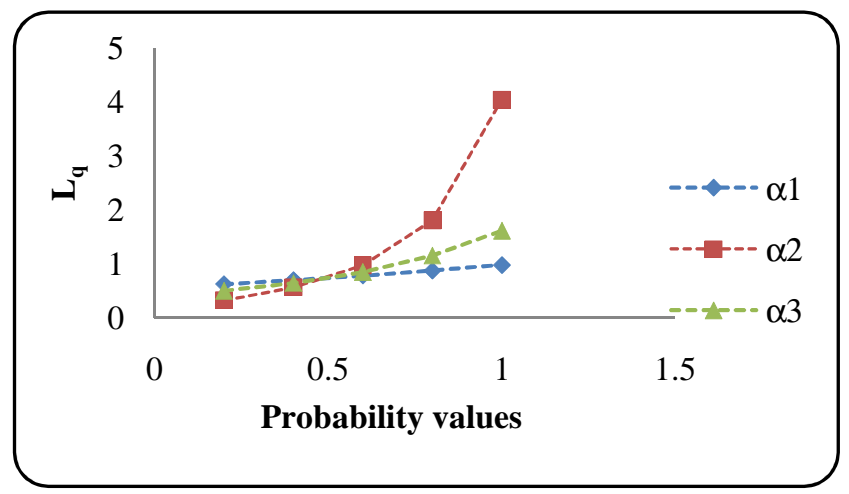

\section{CONCLUSIONS}

Retrial queue with batch arrival admission control and Bernoulli vacation has been investigated in this paper. The necessary and sufficient condition for the system to be stable is obtained. The inputs of the parameters on the performance measures are illustrated.

\section{REFERENCES}

[1] Aissani A 2011 An $\mathrm{M}^{\mathrm{X}} / \mathrm{G} / 1$ Energetic retrial queue with vacation and control IMA Journal of Management Mathematics 22 13-32.

[2] Altman E, Avrachenkov K, Barakat C and Nunez-Queija R 2002 State-dependent M/G/1 type queuing analysis for congestion in control in data networks Comp. Networks39(6) 789-808.

[3] Arivudainambi D and Godhandaraman P 2012 A batch arrival retrial queue with two phase of service, feedback and $\mathrm{K}$ optional vacations Applied Mathematical Sciences6(22) 1071-1087.

[4] Artalejo J R and Li Q 2011 Performance analysis of a block - structured discrete - time retrial queue with state - dependent arrivals Discrete Event Dynamic Systems 20(3) 325-347.

[5] EbenesarAnnabagyam J and UdayaChandrika K 2012 Performance analysis of single server retrial queuing system with Bernoulli and phase type vacations International Journal of Computer Applications54 (7) 30-35.

[6] EbenesarAnnabagyam J and UdayaChandrika K 2010 Single server retrial queuing system with two different vacation policies Int. J. Contemp. Math. Sciences5(32) 1591-1598.

[7] Khalaf R F, Madan K C and Lukas C A 2011 An $\mathrm{M}^{[\mathrm{X}]} / \mathrm{G} / 1$ queue with Bernoulli schedule, general vacation times, random breakdown, general delay times and general repair times Applied Mathematical Sciences5 (1) $35-51$.
[8] Madan K C and Abu-Dayyeh W 2002 Restricted admissibility of batches into an $\mathrm{M}^{\mathrm{X}} / \mathrm{G} / 1$ type bulk queue with modified Bernoulli schedule server vacations ESSAIM : Probability and Statistics6, 113-125.

[9] Madan K C and Choudhury G 2004 Steady state analysis of an $\mathrm{M} /\left(\mathrm{G}_{1}, \mathrm{G}_{2}\right) / 1$ queue with restricted admissibility of arriving batches and modified Bernoulli server vacations under a single vacation policy Journal of Probability and Statistical Sciences2 (2) 167-185.

[10] Prakash Rani K, Srinivasan A and UdayaChandrika K 2008 An M/G/1 retrial queue with additional optional service and server vacation ActaCienciaIndicaXXXIVM (4) 1807-1813.

[11] Srinivasan A, Prakash Rani K and UdayaChandrika K 2007 Retrial queuing system with two-phase service and vacation ActaCienciaIndicaXXXIII M (2) 365-370.

[12] Sumitha D and UdayaChandrika K 2012 Performance

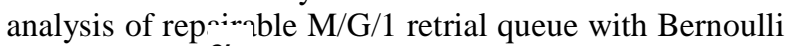
vacation and $\tilde{\alpha}_{3}$ ital search International Journal of Mathematica Archive3 (2).

[13] Sumitha D, EbenesarAnnabagyam J, UdayaChandrika K and Prakash Rani K 2012 Bulk arrival two phase retrial queuing system with orbital search, impatient customers and different types of server vacations International Research Journal1.3 977-987.

[14] UdayaChandrika K, Yasodha D and EbenesarAnnabagyam J 2008 Two phase service retrial queue with emergency and multiple vacations ActaCienciaIndicaXXXIV M (1) 161-168. 